\title{
'Imitation is the Sincerest of Flattery'... Except When It Negatively Impacts Canine and Client Welfare
}

\author{
Gail K. Smith ${ }^{1,2}$ \\ ${ }^{1}$ Department of Clinical Sciences and Advanced Medicine, University \\ of Pennsylvania, Pennsylvania, United States \\ ${ }^{2}$ Antech Imaging Services, Fountain Valley, California, \\ United States \\ Vet Comp Orthop Traumatol 2018;31:311-312.
}

'Imitation is the Sincerest of Flattery. ${ }^{11}$ In a recent published article in VCOT 'Comparison of Three Methods to Quantify Laxity in the Canine Hip' by Broeckx, Vezzoni and colleagues, ${ }^{2}$ the authors claim they developed a 'novel' method to assess hip laxity in dogs. However, the authors' claim is invalid as the method is simply a copy of the well-documented, gold-standard for the measurement of hip laxity, namely, the PennHIP method. Additionally, the 'modified method' by Broeckx, Vezzoni and colleagues lacks essential elements that have made PennHIP globally successful including (1) a non-biased database of genetic information, (2) a network of trained practitioners to perform the procedure with uniformity and (3) a cadre of highly skilled scrutineers to score and critique submitted hip radiographs. Broeckx, Vezzoni and colleagues offer a host of spurious reasons claiming that PennHIP is unpopular in Europe and conclude from the results from testing of a mere 10 dogs in Belgium that European practitioners should abandon PennHIP for the 'Vezzoni-modified Badertscher distension device' method. I am writing on behalf of the several thousand trained PennHIP veterinarians from more than 24 countries who have invested time and effort to join a legitimate and successful science-based programme aimed at reducing the high incidence of canine hip dysplasia.

PennHIP was introduced clinically in 1993 after 10 years of competitively funded research. More than 35 peerreviewed scientific publications validated the PennHIP method and its clinical utility. For more than the past decade, Vezzoni has deceptively claimed that he has developed a 'modified method' to measure hip laxity similar to the PennHIP method but ostensibly based on a different method conceived by Badertscher in a never-published 1977 Masters thesis. ${ }^{3,4}$ In truth, Badertscher's so-called half-axial stress radiographic method positioned the femurs at 45 degrees to
Address for correspondence Gail K. Smith, VMD, PhD, 575 Rock Raymond Road, Downingtown, Pennsylvania 19335, Philadelphia, United States (e-mail: smithgk@vet.upenn.edu).

the radiographic table, a position that our research has shown would show less than $50 \%$ of the laxity revealed with the limbs in the prescribed neutral PennHIP position. ${ }^{4}$ Quoting from Badertscher's thesis, 'The dog was positioned with the femora at approximately a 45-degree angle to the table top....' In contrast to Badertscher's limb position, the 'Vezzoni modified Badertscher distension device method' has the femurs approximately 90 degrees to the radiographic table, ' $\pm 10^{\circ}$ extension, compared with the neutral position'. This positioning is well within the specified PennHIP neutral range as described by Heyman, Smith and colleagues ${ }^{5}$ and contained within USPO no. 5,482,055. The so-called Vezzoni modified Badertscher distension device method is simply a copy and an attempted duplication of the original PennHIP intellectual property (IP) and research, including (1) the limb position to take PennHIP distraction radiographs, (2) the method to measure hip laxity known as the distraction index (DI) and (3) the actual distractor that Vezzoni copied in function if not in exact form. Note the similarity in limb position between a typical PennHIP radiograph submission and Vezzoni's copied method (- Figs. 1 and $\mathbf{2}$ ).

Broeckx, Vezzoni and colleagues also make other misrepresentations. The training and certification process for PennHIP is not costly as claimed. Training has been readily available online and free since 2014. The PennHIP programme is definitively global. It is true that PennHIP charges an evaluation fee and veterinarians await an official report, but this is the modus operandi for all current hip dysplasia control schemes globally. There is no 'obligation' whatsoever that practitioners have digital radiography as claimed. Broeckx and Vezzoni and colleagues claimed savings to veterinarians and pet owners suggesting that the compression view was not necessary in their 10-dog study. However, Broeckx and Vezzoni and colleagues are unaware of received

February 26, 2018

accepted after revision

April 9, 2018

published online

July 4, 2018 (c) 2018 Georg Thieme Verlag KG Stuttgart · New York
DOI https://doi.org/ 10.1055/s-0038-1653961. ISSN 0932-0814. 


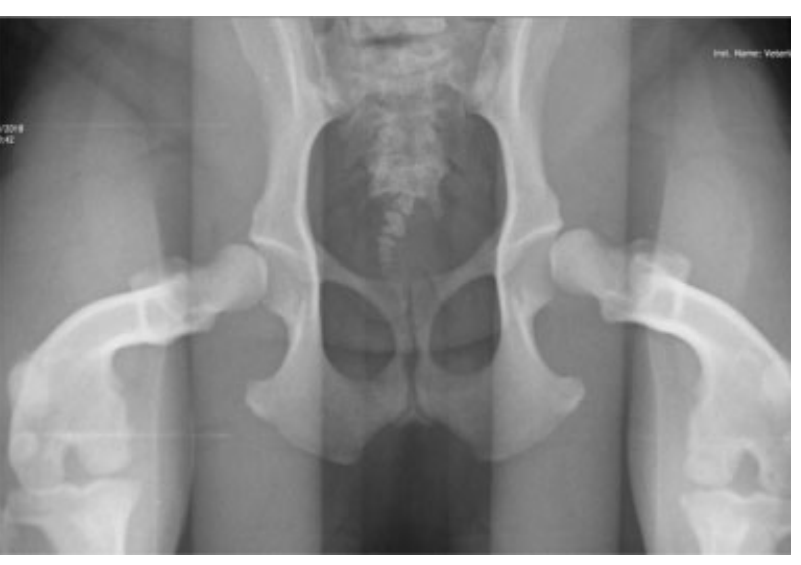

Fig. 1 Distraction position, actual PennHIP case.

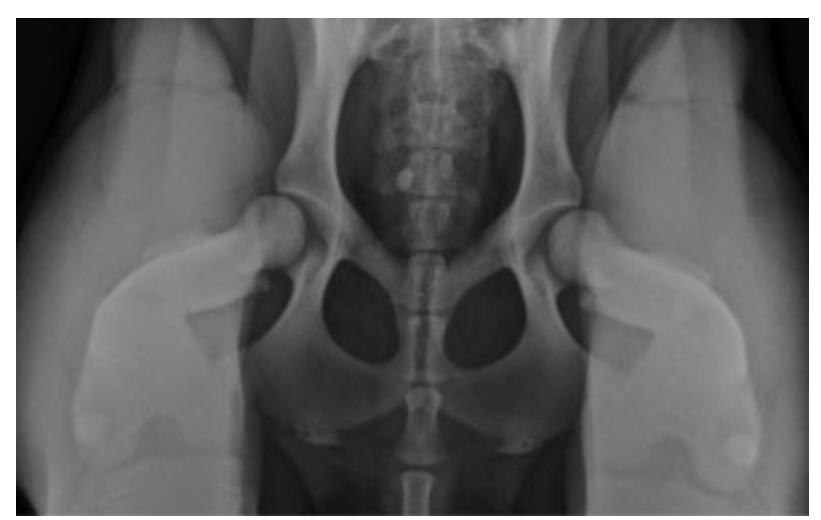

Fig. 2 Vezzoni's duplicated limb position. (Reprinted from Broeckx BJG, Vezzoni A, Bogaerts E, et al. Comparison of three methods to quantify laxity in the canine hip joint. Vet Comp Orthop Traumatol 2018;31(1):23-29.)

proprietary information that we derive from the compression view, information that we no longer share and publish since the early 2000s when Vezzoni began copying the PennHIP IP and radiographic method.

The 'alternative technique' proffered by Broeckx and Vezzoni and colleagues has not been shown by this study of 10 dogs to be as claimed, a 'complete and correct in-house evaluation of the hip joint' for all dogs in Europe. First, the 10 dogs were within a narrow size range. Much greater variability would accompany the inclusion of very large or very small dogs. The authors mentioned 'trained clinicians' were necessary but did not propose who would be 'training' the
European clinicians or how they would be certified. Notably, three of the authors themselves attended formal PennHIP training, likely explaining why their results were so similar to PennHIP's. From our experience it is critical to have trained clinicians performing the PennHIP procedure and equally critical to have specifically trained scrutineers to score and critique the radiographs. The assertion that there will be cost savings with untrained practitioners performing distraction radiography and then reading the images themselves in-hospital is simply not valid and is deceptively misleading. At PennHIP, we fail roughly $10 \%$ of radiographs submitted from trained members worldwide and we provide suggestions for improvements on approximately another $60 \%$ of submitted cases. The practice of untrained and unsupervised clinicians scoring their own radiographs within the veterinary hospital will lead to vastly increased variability of the distraction index in Europe ultimately lowering the value of the metric and wasting the time and money of dog owners and breeders.

This article by Broeckx, Vezzoni and colleagues does not do justice to the large body of research and the three decades required to establish distraction radiography as a legitimate, globally accepted means to evaluate the integrity of the canine hip. The publication does not introduce novel or original ideas, it is devoid of clinical or scientific contribution and it breaches professional ethics. Most troubling, however, is that Broeckx, Vezzoni and colleagues have proposed a short-sighted, self-serving hip dysplasia testing scheme for Europe that is not in the best interests of dogs or dog owners. Dogs deserve better.

\section{References}

1 Charles Caleb Colton. 8th ed. New York: S. Marks \#217; 1824:114

2 Bart J. G. Broeckx, Aldo Vezzoni, Evelien Bogaerts, et al. Comparison of three methods to quantify laxity in the canine hip joint. Vet Comp Orthop Traumatol 2018;31(01):23-29

3 Vezzoni A, Dravelli G, Corbari A, et al. Early diagnosis of canine hip dysplasia. Commissioned Paper in Eur J Comp An Prac 2005;15 (02):173-184

4 Vezzoni A, Dravelli G, Vezzoni L, et al. Comparison of conservative management and juvenile pubic symphysiodesis in the early treatment of canine hip dysplasia. Vet Comp Orthop Traumatol 2008;21(03):267-279

5 Heyman SJ, Smith GK, Cofone MA. Biomechanical study of the effect of coxofemoral positioning on passive hip joint laxity in dogs. Am J Vet Res 1993;54(02):210-215 